\title{
Solution to the Generalized Champagne Problem on Simultaneous Stabilization of Linear Systems *
}

\author{
Qiang Guan ${ }^{1)}$ Long Wang ${ }^{2)} \quad$ Bican Xia $^{3)} \quad$ Lu Yang $^{4)} \quad$ Wensheng Yu ${ }^{1), 5)} \quad$ Zhenbing Zeng ${ }^{4)}$ \\ 1)Laboratory of Complex Systems and Intelligence Science, Institute of Automation, \\ Chinese Academy of Sciences, Beijing 100080, P. R. China. \\ 2) Center for Systems and Control, Department of Mechanics and Engineering Science, \\ Peking University, Beijing 100871, P. R. China. \\ 3)LMAM and School of Mathematical Sciences, Peking University \\ Beijing 100871, P. R. China. \\ 4) Shanghai Institute of Theoretical Computing, Software Engineering Institute, \\ East China Normal University, Shanghai 200062, P. R. China. \\ 5)National Key Laboratory of Intelligent Technology and Systems, \\ Tsinghua University, Beijing 100084, P. R. China.
}

\begin{abstract}
The well-known "Generalized Champagne Problem" on simultaneous stabilization of linear systems is solved by using complex analysis [1, 11, 13, 18, 22] and Blondel's technique [5, 6, 8]. We give a complete answer to the open problem proposed by Patel et al. [20, 21], which automatically includes the solution to the original "Champagne Problem" [6, 8, 9, 17, 20, 21]. Based on the recent development in automated inequality-type theorem proving [31, 32, 33, 35, 36], a new stabilizing controller design method is established. Our numerical examples significantly improve the relevant results in the literature [17, 21].
\end{abstract}

Keywords: linear systems, stabilization, simultaneous stabilization, champagne problem, generalized champagne problem, complex analysis, inequality-type theorem, automated theorem proving.

\section{Introduction}

Simultaneous stabilization of linear systems is a fundamental issue in system and control theory, and is of theoretical as well as practical significance $[5,15,25]$. The basic statement of the simultaneous stabilization problem of linear systems is as follows [5, 15, 23, 25]:

Let $p_{1}, p_{2}, \cdots, p_{k}$ be $k$ scalar linear time-invariant systems. Under what condition does there exist a fixed controller $c$ that is stabilizing for each $p_{i}(i=1, \cdots, k)$ ?

*Supported by National Natural Science Foundation of China (Nos. 60572056, 60528007, 60334020, 60204006, 10471044, and 10372002), National Key Basic Research and Development Program (Nos. 2005CB321902, 2004CB318003, 2002CB312200), the Overseas Outstanding Young Researcher Foundation of Chinese Academy of Sciences and the Program of National Key Laboratory of Intelligent Technology and Systems of Tsinghua University. 
If $k=1$, the above problem is reduced to the stabilization of a single system. There always exists a stabilizing controller for a single system provided no unstable pole-zero cancellations occur $[5$, $15,25]$. Moreover, once a stabilizing controller of a single system is found, it is easy to parametrize the set of all stabilizing controllers of this system [5, 14, 15, 25]. This parametrization is known as Youla-Kucera parametrization discovered by Youla et al. [39, 40] and Kucera [16] respectively.

When $k=2$, by using the Youla-Kucera parametrization, it is possible to rephrase simultaneous stabilization of two systems into strong stabilization (stabilization with a stable controller) of a single system. This relationship was discovered for scaler systems by Saeks and Murray [23], and for multi-variable systems [5, 15, 25] by Vidyasagar and Viswanadham [26]. For the strong stabilization problem of a single system, Youla et al. [38] established an elegant criterion: a system is stabilizable by a stable controller if and only if it has an even number of real unstable poles between each pair of real unstable zeros! This remarkable and easily testable condition is called "parity interlacing property" [5, 15, 25, 38].

For $k \geq 3$, simultaneous stabilization problem is much more complicated than one expected $[5,6,7,8]$. Vidyasagar and Viswanadham [26] stated that it is possible to transform simultaneous stabilization of $k$ systems to strong stabilization of corresponding $k-1$ systems [5, 15, 25]. Blondel et al. [8] proved that simultaneous stabilization of $k$ systems is equivalent to bistable stabilization of associated $k-2$ systems. Bistable stabilization means stabilization with a stable and inverse-stable controller. Such a controller is called bistable controller or unit controller [5]. Although many necessary or sufficient conditions for simultaneous stabilization of three or more systems were obtained in recent years (see [5] and references therein), easily testable necessary and sufficient conditions have not been found yet. Blondel and Gevers [6] showed that the simultaneous stabilization of three systems is not rationally decidable $[5,6]$, i.e., it is not possible to find tractable necessary and sufficient conditions for simultaneous stabilization of three systems that involve only a combination of finite arithmetical operations (addition, substraction, multiplication and division), logical operations ('and' and 'or') and sign test operations (equal to, greater than, greater than or equal to, less than, less than or equal to) on the coefficients of the three systems!

To illustrate the complexity of the simultaneous stabilization problem of three systems, Blondel et al. $[6,8,9]$ proposed a specific simultaneous stabilization problem called "Champagne Problem" where the three systems are explicitly given. Patel [20] solved this problem by showing that there does not exist a stabilizing controller. Furthermore, a more general simultaneous stabilization problem, the "Generalized Champagne Problem", was proposed by Patel et al. [20, 21], which contains the "Champagne Problem" as a special case.

The "Generalized Champagne Problem " is essentially concerned with determining the exact range of a specific parameter in simultaneous stabilization problem [20, 21]. Up to now, there are only some numerical estimates on this range in the literature [17, 21].

It should be pointed out that the "Champagne Problem" and the "Generalized Champagne Problem" are not of much engineering significance in themselves since they both deal with some specific systems. However, by studying these specific problems, we can develop new tools and new methods for general simultaneous stabilization problem. As a matter of fact, by studying some specific systems, Blondel et al. revealed the inherent relationship $[5,6,8,20]$ between the simultaneous stabilization problem and complex analysis theory $[1,11,13,18,22]$, and derived the deep theoretic results of rational undecidability for three systems $[5,6]$.

In this paper, the well-known "Generalized Champagne Problem" on simultaneous stabilization of linear systems is solved by using complex analysis theory [1, 11, 13, 18, 22] and Blondel's technique $[5,6,8]$. We give a complete answer to the open problem proposed by Patel et al. 
$[20,21]$, which automatically includes the solution to the original "Champagne Problem". Based on the recent advances in automated inequality-type theorem proving [31, 32, 33, 36, 35], a novel stabilizing controller design method is established. Our numerical examples significantly improve the relevant results in the literature $[17,21]$.

The paper is organized as follows. We state the generalized champagne problem and give a complete theoretical solution in Section 2. Section 3 deals with the controller design, and some numerical examples are provided. Finally, the conclusion is contained in Section 4.

\section{Solution to the Generalized Champagne Problem}

In this paper, all polynomials are of real coefficients. We denote by $P$ the set of polynomials, $P^{n}$ the set of $n$-th order polynomials where $n$ is an non-negative integer, $H$ the set of Hurwitz stable polynomials (all roots lie within left half of the complex plane), $H^{n}$ the set of $n$-th order Hurwitz polynomials, $S$ the set of Schur stable polynomials (all roots lie outside the unit circle) ${ }^{1}$ and $S^{n}$ the set of $n$-th order Schur polynomials. The variable of polynomials is $s$ or $z$ (we usually use $s$ for Hurwitz polynomials and $z$ for Schur polynomials). Let $C$ denote the complex plane, $D=\{z \in C:|z|<1\}$ be the open unit disc and $\operatorname{cl}(D)=\{z \in C:|z| \leq 1\}$ be the closure of $D$, i.e., the closed unit disc. As usual, $\bar{z}$ stands for the complex conjugate of $z \in C, C_{\infty}=C \cup\{\infty\}$ the extended complex plane and $C_{+\infty}=\{s \in C: \operatorname{Re}(s) \geq 0\} \cup\{\infty\}$ the extended closed right half complex plane.

Throughout this paper, plants and controllers are restricted to single-input single-output systems that are described by linear, time-invariant, real rational transfer functions ${ }^{2}$.

Given a system $p$, a controller $c$ stabilizing $p$ means that the four transfer functions $p c(1+$ $p c)^{-1}, c(1+p c)^{-1}, p(1+p c)^{-1}$ and $(1+p c)^{-1}$ are all stable, i.e., the denominator polynomials of the transfer functions are stable (which means Hurwitz stable in continuous-time case or Schur stable in discrete-time case). The degree of a controller is the maximum of the degrees of its denominator and numerator.

We denote by $U(\operatorname{cl}(D))=\left\{c(z)=\frac{y(z)}{x(z)}: x(z) \in S, y(z) \in S\right\}$ the set of all bistable or unit controllers in discrete time case. Obviously, any element in $U(\operatorname{cl}(D))$ and its inverse are analytic on $\operatorname{cl}(D)$.

The following problem is the well-known "Champagne Problem" $[6,8,9,17,20,21]$ of simultaneous stabilization. It illustrates the difficulty in the simultaneous stabilization of three systems in general. It also shows that, simple problems do not always have simple answers.

Champagne Problem [6, 8, 9, 17, 20, 21] Does there exist a controller that simultaneous

\footnotetext{
${ }^{1}$ Note that, some researchers use the concept of Schur polynomials for polynomials with all roots lying inside unit circle. Most papers on simultaneous stabilization adopt the definition used in this paper, which makes it easier to use the results in complex analysis. It is only a usage and doesn't affect the problem in essence.

${ }^{2}$ Here, the real rational transfer functions are not necessarily proper, that is to say, the degree of numerator may be greater than that of denominator. In the literature on simultaneous stabilization $[5,6,8,9]$, controllers do not have to be proper. It is mainly because that simultaneous stabilization problems can be transformed into the study on the properties of some analytic functions on the extend complex plane [1, 11, 13, 18, 22]. In addition, Blondel [5] gave the conclusion that if $k$ plants are simultaneously stabilizable by a non-proper controller, then they are also simultaneously stabilizable by a proper controller. In fact, once a non-proper controller simultaneously stabilizes $k$ systems, since the roots of a polynomial continuously depend on its coefficients [12, 37], a proper stabilizing controller can be obtained with a sufficiently small perturbation imposed on the denominator polynomial of the original controller. This technique will also be used in the following section on controller design.
} 
stabilizes the following three plants:

$$
p_{1}(s)=\frac{2}{17} \frac{s-1}{s+1}, \quad p_{2}(s)=\frac{(s-1)^{2}}{(9 s-8)(s+1)}, \quad p_{3}(s)=0 ?
$$

Patel [20] solved this problem in 1999 by showing that there does not exist a stabilizing controller. More generally, he considered the following problem:

Generalized Champagne Problem [20,21] What is the range of $\delta$ for the existence of a controller that simultaneously stabilizes the following three plants:

$$
p_{1}(s)=\frac{2 \delta(s-1)}{s+1}, \quad p_{2}(s)=\frac{2 \delta(s-1)^{2}}{((1+\delta) s-(1-\delta))(s+1)}, \quad p_{3}(s)=0 ?
$$

where $\delta$ is a real number.

Obviously, "Generalized Champagne Problem" focuses on determining the range of $\delta$ when there exists a simultaneously stabilizing controller while "Champagne Problem" asks whether $\delta=\frac{1}{17}$ is in this range.

Regarding "Generalized Champagne Problem", as discussed in Patel [20], there does not exist such a controller when $\delta<\frac{1}{16}{ }^{3}$. This conclusion certainly answers "Champagne Problem" and Patel also stated that it remains an open problem [20] whether there exists such a controller when $\delta=\frac{1}{16}$. Leizarowitz et al. $[17,21]$ conjectured the minimum value of $\delta$ for "Generalized Champagne Problem" is $\frac{1}{2 e}=\frac{1}{5.4366}$ and they found a controller for this value of $\delta$. But in 2002, Patel et al. [21] showed that there exists a stabilizing controller $c=\frac{y(s)}{x(s)}$ where $x(s) \in H^{9}, y(s) \in P^{9}$ when $\delta=\frac{1}{6.719367588932806}$. Note that the degree of the controller therein equals 9. Hence, the conjecture mentioned in [17] is invalid. Thus for "Generalized Champagne Problem", it is still an open problem whether there exists a stabilizing controller when $\delta \in\left[\frac{1}{16}, \frac{1}{6.719367588932806}\right)$.

For "Generalized Champagne Problem" with $\delta=\frac{1}{16}$, since the roots of a polynomial continuously depend on its coefficients [12, 37], it is easy to know that there does not exist a controller that simultaneously stabilizes the three plants. Thus we have given an answer to the problem proposed in Patal [20].

It should be pointed out that the above results presented in Patel et al. [21] and Leizarowitz et al. [17] are both based on numerical analysis. The degrees of the controllers provided are high and the conclusions are not theoretically complete.

The following theorem is the main result of this paper which gives a completely theoretical solution to "Generalized Champagne Problem".

Theorem 1 The necessary and sufficient condition for the existence of a controller that simultaneously stabilizes the following three plants:

$$
p_{1}(s)=\frac{2 \delta(s-1)}{s+1}, \quad p_{2}(s)=\frac{2 \delta(s-1)^{2}}{((1+\delta) s-(1-\delta))(s+1)}, \quad p_{3}(s)=0
$$

where $\delta$ is a real number, is $\delta=0$ or $|\delta|>\frac{1}{16}$.

\footnotetext{
${ }^{3}$ In fact, Patel $[20]$ admitted $\delta>0$. Otherwise this condition should be $0<|\delta|<\frac{1}{16}$. Indeed, from the result in the sequel, when $\delta=0$ or $\delta<-\frac{1}{16}$, there does exist a simultaneously stabilizing controller for "Generalized Champagne Problem".
} 
To prove the above theorem, the following two well-known results in complex analysis will be used.

Lemma $1\left(\frac{1}{16}-\right.$ Theorem $)[5,13,18,20]^{4} \quad$ Any analytic function $f$ on $D$ that satisfies:

1) $f(z)=0, z \in D \Leftrightarrow z=0$,

2) $f^{\prime}(0)=1$,

contains an open ball centered at $z=0$ with radius $\frac{1}{16}$ in its range on $D$ but not always a larger ball.

Lemma 2 (Runge's Theorem) $[11,22] \quad$ If $\Omega$ is an open subset of $C$ such that $C_{\infty} \backslash \Omega$ is connected then for each analytic function $f$ on $\Omega$ there is a sequence of polynomials $\left\{q_{n}\right\}$ such that $q_{n}$ uniformly converges to $f$ on any compact subset of $\Omega .{ }^{5}$

We are now in the position to give a proof of Theorem 1.

Proof of Theorem 1 The well known linear fractional transformation $s=\frac{1+z}{1-z}$ is a one-toone mapping of $\operatorname{cl}(D)$ onto $C_{+\infty}$ and its inverse is $z=\frac{s-1}{s+1}$. By this transformation, "Generalized Champagne Problem" is equivalent to the simultaneous stabilization problem of the following three plants in discrete time case:

$$
p_{1 d}(z)=2 \delta z, \quad p_{2 d}(z)=\frac{2 \delta z^{2}}{z+\delta}, \quad p_{3 d}(z)=0,
$$

where $\delta$ is a real number.

The proof contains the following three steps.

Step 1 Firstly, we prove that the three plants defined by Eq. (2) are not simultaneously stabilizable in discrete time case when $0<|\delta|<\frac{1}{16}$.

Assumed by contradiction that, for $0<|\delta|<\frac{1}{16}$, there is a stabilizing controller $c(z)$ for the three plants $p_{1 d}(z), p_{2 d}(z), p_{3 d}(z)$. Then, since $c(z)$ stabilizes $p_{3 d}(z)=0, c(z)$ must be stable. Moreover, $c(z)$ also stabilizes $p_{1 d}(z)=2 \delta z$ and $p_{2 d}(z)=\frac{2 \delta z^{2}}{z+\delta}$ and we have the following equations:

$$
\begin{aligned}
2 \delta z c(z)+1 & =u_{1}(z) \in U(\operatorname{cl}(D)) \\
2 \delta z^{2} c(z)+z+\delta & =u_{2}(z) \in U(\operatorname{cl}(D))
\end{aligned}
$$

Set

$$
f(z)=2 \delta z^{2} c(z)+z=z(2 \delta z c(z)+1) .
$$

Obviously, $f(z)$ is analytic on $D$ and $f(0)=0$ as well as $f^{\prime}(0)=1$. Also, we have

$$
\begin{aligned}
f(z) & =z u_{1}(z) \\
f(z)+\delta & =u_{2}(z)
\end{aligned}
$$

The Eq. (5) means that in $D, f(z)$ is equal to 0 if and only if $z$ is 0 while the Eq. (6) implies that $-\delta$ is not in the range of $f(z)$ in $D$. However, $f(z)$ satisfies the hypothesis of $\frac{1}{16}-$ Theorem, but $0<|\delta|<\frac{1}{16}$. This is impossible, a contradiction is obtained and Step 1 is proved.

Step 2 When $\delta=0$, the three plants defined by Eq.(2) degenerate to a plant $p_{1 d}(z)=$ $p_{2 d}(z)=p_{3 d}(z)=0$. Apparently, $c(z)$ meets the requirement if $c(z)$ is stable.

When $|\delta|=\frac{1}{16}$, since the roots of a polynomial continuously depend on its coefficients $[12,37]$, it is easy to know that there does not exist the desired controller, namely, the three plants defined by Eq. (2) are not simultaneously stabilzable in this case.

\footnotetext{
${ }^{4}$ There is a misprint in [20] for the reference of this lemma where Condition 2 is misprinted as $f(0)=1$.

${ }^{5}$ This lemma is a special case of Runge's Theorem, i.e., a corollary of the original Runge's Theorem [11, 22].
} 
Step 3 We prove that the three plants defined by Eq.(2) are simultaneously stabilizable in discrete time case when $|\delta|>\frac{1}{16}$.

If $|\delta|>\frac{1}{16}$, from Lemma 1 , there exists an analytic function $f(z)$ on $D$ such that $f(z)=$ $0 \Leftrightarrow z=0$ and $f^{\prime}(0)=1$ but $\frac{1}{16} \notin f(D)$. Moreover, $f(z)$ can be a real analytic function, i.e., $f(\bar{z})=\overline{f(z)}{ }^{6}$

Set

$$
g(z)=-16 \delta f\left(\frac{-z}{16 \delta}\right)
$$

According to the properties of $f(z)$, it is obvious that $g(z)$ satisfies the following properties:

1) $g(\bar{z})=\overline{g(z)}$

2) $g(z)$ ia analytic on $|z|<16|\delta|$,

3) $g(z)=0 \Leftrightarrow z=0$, and $g^{\prime}(0)=1$,

4) $g(z)$ leaves out $-\delta$ on $|z|<16|\delta|$.

Let

$$
\mu=\inf _{z \in \operatorname{cl}(D)}|g(z)+\delta|
$$

and

$$
h(z)=\frac{g(z)-z}{2 \delta z^{2}} .
$$

From the above properties of $g(z)$, we have $\mu>0, h(z)$ is analytic on $|z|<16|\delta|$, and

$$
1+2 \delta z h(z) \neq 0, \quad z \in \operatorname{cl}(D)
$$

By Runge's Theorem, there exists a real polynomial $q(z)$ such that

$$
|h(z)-q(z)|<\frac{\mu}{2|\delta|}\left(\frac{1}{16 \delta}\right)^{2}, \quad z \in \operatorname{cl}(D) .
$$

Since $h(z)$ satisfies $(10), q(z)$ also satisfies

$$
1+2 \delta z q(z) \neq 0, \quad z \in \operatorname{cl}(D)
$$

i.e.,

$$
1+2 \delta z q(z)=u_{1}(z) \in U(\operatorname{cl} D) .
$$

By the definition of $h(z)$, we have

$$
\left|g(z)-z-2 \delta z^{2} q(z)\right|<\mu, \quad z \in \operatorname{cl}(D) .
$$

Set

$$
p(z)=z+2 \delta z^{2} q(z)
$$

Obviously, $p(0)=0, p^{\prime}(0)=1$. By the definition of $\mu$,

$$
p(z) \neq-\delta, \quad z \in \operatorname{cl}(D)
$$

Hence, we have

$$
\delta+z+2 \delta z^{2} q(z)=u_{2}(z) \in U(\operatorname{cl} D) .
$$

\footnotetext{
${ }^{6}$ Special constructions of such functions are presented in $[13,18]$, for instance,

$$
f(z)=z \prod_{n=1}^{\infty}\left(\frac{1+z^{2 n}}{1+z^{2 n-1}}\right)^{8} .
$$
}


Let $c(z)=q(z)$, by (13) and (17), $c(z)$ is the desired controller. That completes the proof of Step 3.

Theorem 1 is now proved by combining Steps 1-3.

Remark 1 Theorem 1 contains a complete theoretical answer to the "Generalized Champagne Problem" [20, 21], automatically including the solution to the "Champagne Problem" [6, 8, 9, 20]. The main result in Patel [20] is also contained in Step 1 of the proof of Theorem 1. Apparently, the proof in this paper is much more concise and straightforward than that in Patel [20].

Remark 2 As a byproduct, Step 2 of the proof of Theorem 1 contains an answer to an open problem proposed in Patel [20].

By a similar argument as in the proof of Theorem 1, we have

Theorem 2 The necessary and sufficient condition for the existence of a controller that simultaneously stabilizes the following three plants in continuous time case:

$$
p_{1}(s)=\frac{s-1}{s+1}, \quad p_{2}(s)=\frac{(s-1)^{2}}{\left((1-\delta) s^{2}-2 \delta s-(1+\delta)\right.}, \quad p_{3}(s)=0,
$$

where $\delta$ is a real number, or, equivalently, simultaneously stabilizes the following three plants in discrete time case:

$$
p_{1 d}(z)=z, \quad p_{2 d}(z)=\frac{z^{2}}{z-\delta}, \quad p_{3 d}(z)=0,
$$

where $\delta$ is a real number, is $\delta=0$ or $|\delta|>\frac{1}{16}$.

Remark 3 Some partial results of Theorem 2 are available in [5, 7, 8]. In [5], a sufficient condition was given on the nonexistence of a simultaneously stabilizing controller for the three plants (in continuous time case) in Theorem 2.

Remark 4 It should be pointed out that Theorem 2 is equivalent to Theorem 1 in some sense. In fact, a stabilizing controller multiplied by a non-zero constant is also a stabilizing controller. When $\delta \neq 0$, by multiplying the three plants in (19) by $-2 \delta$, and letting $\delta_{1}=-\delta$, it is obvious that the three plants obtained are the same as the three plants in Theorem 1.

Remark 5 Although a complete theoretical solution to the "Generalized Champagne Problem" is provided in Theorem 1, it can be seen from the proof of Theorem 1 that it is still difficult in practice to construct the simultaneously stabilizing controller. This difficulty can also be seen by the controller design examples in [17, 21]. A novel stabilizing controller design method is established in the next section, and the numerical examples obtained significantly improve the relevant results in $[17,21]$.

\section{Controller Design}

Although the "Generalized Champagne Problem" has been theoretically resolved, it is still difficult in practice to construct the simultaneously stabilizing controller. This fact can also be seen in the specific controller design examples in $[17,21]$. In particular, how can one construct the simultaneously stabilizing controller with minimal degree for $\delta>\frac{1}{16}$ ? And when the degree of the controller is fixed, how to determine the range of $\delta$ ? These issues are of both theoretical and practical significance.

When the degree of the controller is fixed, the controller design problem of simultaneous stabilization can be transformed in essence to the problem of how to solve a set of algebraic inequalities. Early in 1950s Tarski published the well-known work [24] on the decidability [2, 24, 27, 28, 30, 37] of this kind of problems. Tarski's decision algorithm is of theoretical significance only, since it can 
not be used to verify any non-trivial algebraic or geometric propositions in practice $[27,28,30,37]$ due to its very high computational complexity. The Cylindrical Algebraic Decomposition (CAD) $[3,4,10]$ algorithm proposed by Collins and subsequently improved by him and his collaborators is the first practical decision algorithm and can be used to verify non-trivial algebraic or geometric propositions on computer. Although, as a generic program for automated theorem proving, its computational complexity was still very high, the CAD and its improved variations have become one of the major tools for solving this kind of problems.

Wu [27] proposed in 1977 a new decision procedure for proving geometry theorems. As an important progress in automated theorem proving [28, 30, 37], Wu's method is very efficient for mechanically proving elementary geometry theorems of equality type. The success of Wu's method has inspired in the world the research of algebraic approach to automated theorem proving [28, 30,37 . However, automated inequality proving has been a difficult topic in the area of automated reasoning for many years since the relevant algorithms depend on real algebra and real geometry. In 1996, Yang and his colleagues [34, 37] introduced a powerful tool, the Complete Discrimination Systems (CDS) of Polynomials, for automated reasoning in real algebra. By means of CDS, many inequality-type theorems from various applications have been proved or discovered.

In recent years, by combining discriminant sequences of polynomials [34, 37] with Wu's method $[27,28,30]$ as well as Partial Cylindrical Algebraic Decomposition [3, 4, 10], Yang et al. proposed some algorithms which are able to discover new inequalities. These algorithms are complete for an extensive class of problems involving inequalities and are applicable to the controller design in simultaneous stabilization. Based on these algorithms, a generic program called "Discoverer" $[34,35]$ and a generic program called "Bottema" [31, 32, 36] were implemented as Maple packages. The following controller examples are all obtained automatically by calling "Discoverer" [34, 35] or "Bottema" [31, 32, 36].

The following sufficient condition for checking the Hurwitz stability of a polynomial may be used for improving computational efficiency when calling the above programs.

Lemma $3[19] \quad$ Suppose $f(s)=a_{0}+a_{1} s+\cdots+a_{n} s^{n}\left(a_{i}>0, i=0,1,2, \cdots, n, n \geq 3\right), f(s)$ is Hurwitz stable if

$$
a_{i-1} a_{i+2} \leq 0.4655 a_{i} a_{i+1}, \quad(i=1,2, \cdots, n-2) .
$$

In addition, to get a proper controller, we introduce the following theorem on the disturbance to the Hurwitz polynomials.

Theorem 3 Given a polynomial $f(s)=a_{0}+a_{1} s+\cdots+a_{m} s^{m}\left(a_{i}>0, i=0,1,2, \cdots, m\right) \in$ $H^{m}$, then for any integer $n>m$ and sufficiently small $\varepsilon>0$, there exist $\varepsilon_{i}, 0<\varepsilon_{i}<\varepsilon(i=$ $m+1, m+2, \cdots, n)$, such that $g(s)=a_{0}+a_{1} s+\cdots+a_{m} s^{m}+\varepsilon_{m+1} s^{m+1}+\varepsilon_{m+2} s^{m+2}+\cdots+\varepsilon_{n} s^{n} \in H^{n}$.

Proof Suppose $f(s)=a_{0}+a_{1} s+\cdots+a_{m} s^{m}\left(a_{i}>0, i=0,1,2, \cdots, m\right) \in H^{m}$, we only need to prove that $g(s)=a_{0}+a_{1} s+\cdots+a_{m} s^{m}+\varepsilon s^{m+1} \in H^{m+1}$ for sufficiently small $\varepsilon>0$ because we can treat $g(s)$ with the same manner iteratively.

Since $f(s)=a_{0}+a_{1} s+\cdots+a_{m} s^{m} \in H^{m}$, it is well known that Hurwitz principal minors $\Delta_{i}^{f}>0(i=1,2, \cdots, m)[12]$.

By computing Hurwitz principal minors of $g(s)=a_{0}+a_{1} s+\cdots+a_{m} s^{m}+\varepsilon s^{m+1}$, it is easy to verify that $\Delta_{1}^{g}=a_{m}, \Delta_{i}^{g}=a_{m} \Delta_{i-1}^{f}+\varepsilon \delta_{i}\left(\varepsilon, a_{0}, a_{1}, \cdots, a_{m}\right)(i=2, \cdots, m, m+1)$, where $\delta_{i}\left(\varepsilon, a_{0}, a_{1}, \cdots, a_{m}\right)(i=2, \cdots, m, m+1)$ are polynomials in $\left(\varepsilon, a_{0}, a_{1}, \cdots, a_{m}\right)$. Obviously, when $\varepsilon>0$ is sufficiently small, we have $\Delta_{i}^{g}>0(i=1,2, \cdots, m, m+1)$. This completes the proof.

In what follows, we will provide some controller examples for "Generalized Champagne Problem" of simultaneous stabilization by applying "Discoverer" [34, 35] or "Bottema" [31, 32, 36]. 
Without loss of generality, we suppose the denominator polynomials of the controllers are monic. In addition, for convenience, we only consider the case $\delta>0$.

Example 1 For the three plants defined in "Generalized Champagne Problem", when the degree of controllers is restricted to 0 , i.e., the controllers are constants, invoking "Discoverer" or "Bottema", we obtain the following results. There exist desired controllers for $\delta>\frac{1}{2}$, whereas there do not exist requested controllers for $\delta \leq \frac{1}{2}$. For a given $\delta>\frac{1}{2}$, for example, $\delta=\frac{3}{4}, c(s)=y_{0}$ is a desired controller if and only if $\frac{1}{6}<y_{0}<\frac{1}{2}$.

Remark 6 Example 1 looks simple, but it shows that: 1) When the degree of the controller is restricted, the explicit bound of $\delta$ can be obtained by using the packages developed by Yang et al.; 2) Moreover, when $\delta$ is fixed, the ranges of the parameters of controllers can be obtained. Due to the completeness of these algorithms, the conditions obtained are both necessary and sufficient.

Example 2 For the three plants defined in "Generalized Champagne Problem", when the numerator polynomials of controllers are restricted to 0 -th order polynomials and the denominator polynomials of controllers to 1st order polynomials, i.e., the controllers have the form $c(s)=$ $\frac{y_{0}}{s+x_{0}}$, invoking "Discoverer" or "Bottema", we have the following results as in the above example. There exist desired controllers for $\delta>\frac{1}{2}$, whereas there do not exist requested controllers for $\delta \leq \frac{1}{2}$. For a given $\delta>\frac{1}{2}$, say, $\delta=\frac{3}{4}, c(s)=\frac{1}{s+3}$ is a desired controller.

Example 3 For the three plants defined in "Generalized Champagne Problem", when the numerator polynomials of controllers are restricted to 1 st order polynomials and the denominator polynomials of controllers to 0 - th order polynomials, i.e., the controllers have the form $c(s)=$ $y_{1} s+y_{0}$, invoking "Discoverer" or "Bottema", we have the following results. There exist desired controllers for $\delta>\frac{1}{4}$, whereas there do not exist requested controllers for $\delta \leq \frac{1}{4}$. For a given $\delta>\frac{1}{4}$, say $\delta=\frac{1}{2}$, if $\left(y_{1}, y_{0}\right) \in\left\{\left(\frac{1}{10}, \frac{51}{100}\right),\left(\frac{3}{5}, \frac{3}{5}\right),\left(\frac{4}{5}, \frac{3}{5}\right)\right\}, c(s)=y_{1} s+y_{0}$ is a requested controller. Moreover, to get a proper controller, by the continuous dependance for roots of polynomials on their coefficients [12, 37] and Theorem 3, if $\left(x_{1}, y_{1}, y_{0}\right) \in\left\{\left(\varepsilon, \frac{1}{10}, \frac{51}{100}\right),\left(\varepsilon, \frac{3}{5}, \frac{3}{5}\right),\left(\varepsilon, \frac{4}{5}, \frac{3}{5}\right)\right\}$ and $\varepsilon>0$ is sufficiently small, e.g., $\varepsilon=\frac{1}{10}, \widetilde{c}(s)=\frac{y_{1} s+y_{0}}{\varepsilon s+1}$ is a desired proper controller.

Example 4 For the three plants defined in "Generalized Champagne Problem", when the numerator polynomials of controllers are restricted to 1 st order polynomials and the denominator polynomials of controllers to 1 st order polynomials, i.e., the controllers have the form $c(s)=$ $\frac{y_{1} s+y_{0}}{s+x_{0}}$, invoking "Discoverer" or "Bottema", we have that there exist desired controllers for $\delta>\frac{1}{4}$ whereas there do not exist requested controllers for $\delta \leq \frac{1}{4}$. For a given $\delta>\frac{1}{4}$, say, $\delta=\frac{1}{3}$, if $\left(x_{0}, y_{1}, y_{0}\right) \in\left\{\left(2, \frac{31}{10}, \frac{201}{100}\right),\left(3, \frac{41}{10}, \frac{301}{100}\right),\left(6,8, \frac{61}{10}\right)\right\}, c(s)=\frac{y_{1} s+y_{0}}{s+x_{0}}$ is a desired controller.

Remark $7 \quad$ The controllers obtained in the above examples are the sample points picked out from the cells of some appropriate decomposition of the parametric space which satisfy the requirements of simultaneous stabilization. As mentioned in Example 1, when the degrees of controllers are restricted, the explicit bound of $\delta$ and the ranges of the parameters of the controllers can be obtained by further analysis on the distribution of the roots of the polynomials output by "Discoverer".

Example 5 For the three plants defined in "Generalized Champagne Problem", when the numerator polynomials of controllers are restricted to 2 nd order polynomials and the denominator polynomials of controllers to 0 - th order polynomials, i.e., the controllers have the form $c(s)=$ $y_{2} s^{2}+y_{1} s+y_{0}$, invoking "Discoverer", we have that there do not exist desired controllers for 
$\delta=\frac{1}{6}$. When $\delta=\frac{10}{59}$, using the sufficient condition proposed in Lemma 3 for Hurwitz stability, we have that there exist such kind of controllers. For example, if $\left(y_{2}, y_{1}, y_{0}\right) \in\left\{\left(\frac{191}{100}, \frac{39001}{10000}\right.\right.$,

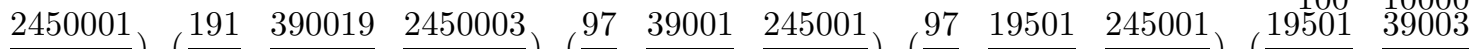
$\left.\frac{2450001}{1000000}\right),\left(\frac{191}{100}, \frac{350019}{100000}, \frac{2450003}{1000000}\right),\left(\frac{97}{50}, \frac{35001}{10000}, \frac{245001}{100000}\right),\left(\frac{97}{50}, \frac{19501}{5000}, \frac{245001}{100000}\right),\left(\frac{19501}{10000}, \frac{39003}{10000}\right.$, $\left.\left.\frac{4900299}{2000000}\right)\right\}, c(s)=y_{2} s^{2}+y_{1} s+y_{0}$ is a desired controller. Moreover, to get a proper controller, by the continuous dependance for roots of polynomials on their coefficients [12, 37] and Theorem 3, if $\left(x_{2}, x_{1}, y_{2}, y_{1}, y_{0}\right) \in\left\{\left(\varepsilon, \varepsilon, \frac{191}{100}, \frac{39001}{10000}, \frac{2450001}{100000}\right),\left(\varepsilon, \varepsilon, \frac{191}{100}, \frac{390019}{100000}, \frac{2450003}{1000000}\right),\left(\varepsilon, \varepsilon, \frac{97}{50}, \frac{39001}{10000}\right.\right.$, $\left.\left.\frac{245001}{100000}\right),\left(\varepsilon, \varepsilon, \frac{97}{50}, \frac{19501}{5000}, \frac{245001}{100000}\right),\left(\varepsilon, \varepsilon, \frac{19501}{10000}, \frac{39003}{10000}, \frac{4900299}{2000000}\right)\right\}$ and $\varepsilon>0$ is sufficiently small, e.g., $\varepsilon=\frac{1}{10000000}$, then $\widetilde{c}(s)=\frac{y_{2} s^{2}+y_{1} s+y_{0}}{\varepsilon s^{2}+\varepsilon s+1}$ is a desired proper controller.

Remark 8 The value of $\delta$ obtained in Example 5 is an improvement over the bound conjectured in [17]. Although an improvement over this bound was also made by Patel et al. [21], the degree of the controller provided by them equals 9 whereas the controller presented in Example 5 is of degree 2 which is much more lower. In addition, we can see from the above examples that the improvement over the bound of $\delta$ conjectured in [17] could not be achieved by controllers with degrees less than 2 .

Example 6 For the three plants defined in "Generalized Champagne Problem", suppose the numerator polynomials of controllers are restricted to $3 \mathrm{rd}$ order polynomials and the denominator polynomials of controllers to $0-$ th order polynomials, i.e., the controllers have the form $c(s)=y_{3} s^{3}+y_{2} s^{2}+y_{1} s+y_{0}$. When $\delta=\frac{1}{7}$, using the sufficient condition proposed in Lemma 3 for Hurwitz stability, we have that there exist such kind of controllers. For example, if $\left(y_{3}, y_{2}, y_{1}, y_{0}\right) \in\left\{\left(\frac{1037}{1000}, \frac{30077}{10000}, \frac{50001}{10000}, \frac{300001}{100000}\right),\left(\frac{26}{25}, \frac{376}{125}, \frac{50001}{10000}, \frac{300001}{100000}\right),\left(\frac{113}{100}, \frac{378}{125}, \frac{50001}{10000}\right.\right.$, $300001,57029 \quad 121,50001,300001)\left(\begin{array}{lllllll}11407 & 121 & 50001 & 300001\end{array}\right),\left(\begin{array}{lllll}114113 & 1513 & 50001\end{array}\right.$ $\frac{100000}{100},\left(\frac{57000}{50000}, \frac{12}{40}, \frac{5000}{10000}, \frac{30000}{10000}\right)\left(\frac{1100}{10000}, \frac{12}{40}, \frac{5000}{10000}, \frac{30000}{100000}\right),\left(\frac{11113}{100000}, \frac{1513}{500}, \frac{5001}{10000}\right.$, $\left.\left.\frac{300001}{100000}\right)\right\}, c(s)=y_{3} s^{3}+y_{2} s^{2}+y_{1} s+y_{0}$ is a desired controller. Moreover, to get a proper controller, by the continuous dependance for roots of polynomials on their coefficients [12, 37] and Theorem 3, if $\left(x_{3}, x_{2}, x_{1}, y_{3}, y_{2}, y_{1}, y_{0}\right) \in\left\{\left(\varepsilon_{1}, \varepsilon, \varepsilon, \frac{1037}{1000}, \frac{30077}{10000}, \frac{50001}{10000}, \frac{300001}{100000}\right),\left(\varepsilon_{1}, \varepsilon, \varepsilon, \frac{26}{25}, \frac{376}{125}, \frac{50001}{10000}\right.\right.$, $\left.\frac{300001}{100000}\right),\left(\varepsilon_{1}, \varepsilon, \varepsilon, \frac{113}{100}, \frac{378}{125}, \frac{50001}{10000}, \frac{300001}{100000}\right),\left(\varepsilon_{1}, \varepsilon, \varepsilon, \frac{57029}{50000}, \frac{121}{40}, \frac{50001}{10000}, \frac{300001}{100000}\right),\left(\varepsilon_{1}, \varepsilon, \varepsilon\right.$, $\left.\left.\frac{11407}{10000}, \frac{121}{40}, \frac{50001}{10000}, \frac{300001}{100000}\right),\left(\varepsilon_{1}, \varepsilon, \varepsilon, \frac{114113}{100000}, \frac{1513}{500}, \frac{50001}{10000}, \frac{300001}{100000}\right)\right\}$ and $\varepsilon^{2}>\varepsilon_{1}$ for sufficiently small $\varepsilon_{1}>0$ and $\varepsilon>0$, e.g., $\varepsilon=10^{-7}$ and $\varepsilon_{1}=10^{-15}, \widetilde{c}(s)=\frac{y_{3} s^{3}+y_{2} s^{2}+y_{1} s+y_{0}}{\varepsilon_{1} s^{3}+\varepsilon s^{2}+\varepsilon s+1}$ is a desired proper controller.

Remark 9 The value of $\delta$ appeared in Example 6 is an improvement over the minimal bound proposed in [21]. The degree of the controller presented in [21] equals 9 whereas the controller given in Example 6 is of degree 3 which is much more lower.

Remark 10 The above algorithms are also complete for high-order controller design. From our computational experiments, other phenomena can be observed. For instance, the improvement on the bound of $\delta$ in "Generalized Champagne Problem" mainly depends on the increase on the order of numerator polynomial of the stabilizing controller. These problems deserve further research and are omitted here for succinctness.

Remark 11 The generic programs "Discoverer" [33, 35] and "Bottema" [31, 32, 36] are powerful tools in practice for automated inequalities proving and can be applied in various fields. In this paper, only some basic functions of those packages are employed to determine the ranges of parameters and to design simultaneously stabilizing controllers. In previous examples, it is demon- 
strated how powerful these packages are. Indeed, according to the Finiteness Theorem proposed by W.T. Wu [29] on global-optimization, global-optimization problems can be theoretically solved by 'Discoverer" [33, 35] and "Bottema" [31, 32, 36] where the objectives are polynomials and constraints are also polynomial equalities or inequalities. The potential applications of the packages are considerable.

Remark 12 It should be pointed out that, although "Discoverer" [33, 35] and Bottema [31, 32, 36] are powerful, the computational complexity increases very quickly with the dimension, i.e., the number of parameters. It is a problem deserving further study how to promote the efficiencies of these algorithms for dealing with the large-scale engineering optimization problems. Combining symbolic computation with numerical calculation as well as large-scale parallel numerical processing may be an effective way [30].

\section{Conclusion}

In this paper, the well-known "Generalized Champagne Problem" for simultaneous stabilization of linear systems has been resolved by using complex analysis [1, 11, 13, 18, 22] and Blondel's technique $[5,6,8]$. We gave a complete answer to the open problem proposed in Patel et al. $[20,21]$, which automatically includes the solution to the original "Champagne Problem" $[6,8$, $9,17,20,21]$. Based on the recent developments in automated inequality-type theorem proving $[31,32,33,36,35]$, a novel stabilizing controller design method has been established. Our numerical examples significantly improved the relevant results in the literature [17, 21].

\section{Acknowledgements}

The authors express their thanks to Prof Wenjun Yuan of Guangzhou Univ and Dr Nong Gu of Dekin Univ in Australia for providing some relevant papers.

\section{References}

[1] L. Ahlfors, Conformal Invariants: Topics in Geometric Function Theory (McGraw-Hill Series in Higher Mathematics), McGraw-Hill Book Company, New York, 1973.

[2] B. Anderson, N. Bose, and E. Jury, Output feedback stabilization and related problems solution via decision methods, IEEE Transactions Automatic Control, 20(1): 53-66, 1975.

[3] D. S. Arnon, G. E. Collins, and S. McCallum, Cylindrical algebraic decomposition I: the basic algorithm, SIAM Journal on Computing, 13(4): 865-877, 1984.

[4] D. S. Arnon, G. E. Collins, and S. McCallum, Cylindrical algebraic decomposition II: an adiacency algorithm for the plan, SIAM Journal on Computing, 13(4): 878-889, 1984.

[5] V. Blondel, Simultaneous Stabilization of Linear Systems (Lecture Notes in Control and Information Sciences 191), Springer-Verlag, London, 1994.

[6] V. Blondel and M. GeversSimultaneous stabilization of three linear systems is rationally undecidable Mathematics of Control, Signals, and Systems, 6(2): 135-145, 1993. 
[7] V. Blondel, M. Gevers, R. Mortini, and R. Rupp, Stabilizable by a stable and by an inverse stable but not by a stable and inverse atable, Proceedings of the 31st Conference on Decision and Control, Tucson, Arizons, 832-833, 1992.

[8] V. Blondel, M. Gevers, R. Mortini, and R. Rupp, Simultaneous stabilization of three or more systems: Conditions on the real axis do not suffice, SIAM Journal on Control and Optimization, 32(2): 572-590, 1994.

[9] V. Blondel, E. D. Sontag, M. Vidyasagar, and J. C. Willems, Open Problems in Mathematical Systems and Control Theory, Springer, London, 1999.

[10] G. E. Collins and H. Hong, Partial cylindrical algebraic decomposition for quantifier elimination, Journal of Symbolic Computation, 12(3): 299-328, 1991.

[11] J. Conway, Functional of One Complex Variable (Second Edition), Springer-Verlag, New York, 1978.

[12] F. Gantmacher, Matrix Theory, Chelsea, New York, 1959.

[13] G. Glouzin, Geometric Theory of functions of a complex variable, Translation of Math. Monographs, vol. 26, American Math. society, 1969.

[14] C. Desoer, R. Liu, J. Murray, and R. Seaks, Feedback system design: the fractional representation approach to analysis and synthesis, IEEE Transactions Automatic Control, 25(3): 399-412, 1980.

[15] J. Doyle, B. Francis, A. Tannenbaum, Feedback Control Theory. Macmillan Publishing Co., New York, 1991.

[16] V. Kucera, Discrete Linear Control: The Polynomial Equation Approach, Wiley, New York, 1979 .

[17] A. Leizarowitz, J. Kogan, and E. Zeheb, On simultaneous stabilization of linear plants, Latin American Applied Research, 29(3-4): 167-174, 1999.

[18] Z. Nehari, Conformal Mapping. McGraw-Hill Book Company, Inc., New York, 1952.

[19] Y. Y. Nie, Criteria for the stability of polynomials (in Chinese), Mechanics, (2): 110-116, 1976.

[20] V. V. Patel, Solution to the Champagne Problem on the simultaneous stabilization of three plants, Systems and Control Letters, 37(3): 173-175, 1999.

[21] V. V. Patel, G. Deodhare, and T. Viswanath, Some applications of randomized algorithms for control system design, Automatica, 38(12): 2085-2092, 2002.

[22] W. Rudin, Real and Complex Analysis (Third Edition), McGraw-Hill Companies, Inc., New York, 1987.

[23] R. Saeks and J. Murray, Fractional representation, algebraic geometry and the simultaneous stabilization problem, IEEE Transactions Automatic Control, 27(4): 895-903, 1982.

[24] A. Tarski, A Decision Method for Elementary Algebra and Geometry. The University of California Press, Berkeley, 1951. 
[25] M. Vidyasagar, Control System Synthesis: A Factorization Approach. The MIT Press, Cambridge, Massachusetts, London, 1985.

[26] M. Vidyasagar and N. Viswanadham, Algebraic design techniques for reliable stabilization, IEEE Transactions Automatic Control, 27(5): 1085-1095, 1982.

[27] W. T. Wu, On the decision problem and mechanization of theorem-proving in elementarygeometry, Science in China, (21): 159-172, 1978.

[28] W. T. Wu, Mechanical Theorem Proving in Geometries: Basic Principles, (translated from the Chinese by X. Jin and D. Wang), Springer, New York, 1984.

[29] W. T. Wu, On a finiteness theorem about problem involving inequalities, System Science and Mathematical Science, 7(2): 193-200, 1994.

[30] W. T. Wu, Mathematics mechanization (Series in Mathematics Mechanization), Science Press, Beijing, 2003. (in Chinese)

[31] L. Yang, A dimension-decreasing algorithm with with generic program foe aotomated inequalities proving, High Technology Letters, 8(7): 20-25, 1998. (in Chinese)

[32] L. Yang, Recent advances in automated theorem proving on inequalities, Journal of Computer Science and Technology, 14(5): 434-446, 1999.

[33] L. Yang, X. R. Hou, B. C. Xia, A complete algorithm for automated discovering of a class of inequality-type theorems Science in China, F-44(1): 33-49, 2001.

[34] L. Yang, X. R. Hou, and Z. B. Zeng, A complete discrimination system for polynomials, Science in China, E-39(6): 628-646, 1996.

[35] L. Yang, B. C. Xia, Real solution classifications of a class of parametric semi-algebraic systems, Algorithmic Algebra and Logic Proceedings of the A3L 2005 (A. Dolzmann, A. Seidl, and T. Sturm, eds.), 281-289, Herstellung und Verlag, Norderstedt, 2005.

[36] L. Yang, S. H. Xia, Automated proving for a class of constructive geometric inequalities, Chinses Journal of Computers, 26(7): 769-778, 2003. (in Chinses)

[37] L. Yang, J. Z. Zhang, and X. R. Hou, Non-linear equations systems and automated theorm proving (Series in Nonlinear Sciences), Shanghai Press of Science, Technology, and Education, Shanghai, 1996. (in Chinses)

[38] D. Youla, J. Bongiorno, and C. Lu, Single-loop feedbak stabilization of linear multivariable plants, Automatica, 10(2): 159-173, 1974.

[39] D. Youla, J. Bongiorno, and H. Jabr, Modern Wiener-Hopf design of optimal controllers - part I: the single-input-output case, IEEE Transactions Automatic Control, 21(1): 3-13, 1976.

[40] D. Youla, H. Jabr, and J. Bongiorno, Modern Wiener-Hopf design of optimal controllers - part II: the multivariable case, IEEE Transactions Automatic Control, 21(3): 319-338, 1976. 